\title{
Editorial
}

\section{Biomarkers for Psychiatric Disorders: Where Are We Standing?}

\author{
Daniel Martins-de-Souza ${ }^{1,2,3}$ \\ ${ }^{1}$ Research Group of Proteomics, Department of Psychiatry and Psychotherapy, Ludwig Maximilians University (LMU), \\ Nussbaumstraße 7, 80336 Munich, Germany \\ ${ }^{2}$ Research group of Proteomics and Biomarkers, Max Planck Institute of Psychiatry, Munich, Germany \\ ${ }^{3}$ Laboratorio de Neurociências (LIM-27), Instituto de Psiquaitria, Faculdade de Medicina da Universidade de São Paulo, \\ SP, São Paulo, Brazil \\ Correspondence should be addressed to Daniel Martins-de-Souza; danms90@gmail.com
}

Received 16 April 2013; Accepted 18 April 2013

Copyright (c) 2013 Daniel Martins-de-Souza. This is an open access article distributed under the Creative Commons Attribution License, which permits unrestricted use, distribution, and reproduction in any medium, provided the original work is properly cited.

The answer for the question posed in the title is: considering a clinical applicability, we are standing in the very beginning. To date, there are no biomarkers of any kind available to any of the psychiatric disorders, and perhaps establishing those will be one of the most difficult tasks that medical scientists will ever face, This is due to several reasons: (1) the multifactorial characteristic of psychiatric disorders, (2) these are multigenic disorders in which each gene has a small effect; (3) the environment exerts a heavy influence in the establishment of the disease. These reasons lead us to conclude that "the biomarker" for a psychiatric condition will never exist. At best, we will be able to identify panels or sets of biomarkers.

Each psychiatric condition is a heterogeneous entity: for example, bipolar disorder is one disease composed by very opposite phases and symptoms, if we consider maniac and depressive episodes. Moreover, there is a significant overlap of symptoms among all psychiatric disorders. It has been shown that up to $31 \%$ of the bipolar disorder patients may be initially diagnosed with schizophrenia [1]. Still in this line, there have been concerns about false-positive clinical diagnosis due to the subjective criteria differently adopted among clinicians [2], supporting the necessity of establishing molecular biomarkers that could at least guide a clearer diagnostic decision. Nevertheless, at the current stage, diagnostic biomarkers do not seem to be the most needed ones. First, experienced clinicians have a good notion about the diagnosis, even with all concerns about subjectivity. Also, patients suffer considerably with the uneven kind of treatments available, making medication biomarkers more important at this particular point of psychiatric research. Furthermore, establishing diagnostic biomarkers at the disease onset is not a trivial task. Recently, the first and only diagnostic test proposed for schizophrenia [3], the VeriPsych, was discontinued supporting this notion (http://www.veripsych.com/).

The most needed type of biomarkers to be applicable in a short-term future are those which could predict or indicate the likelihood of a successful treatment. The "one treatment fits all" notion it is not applicable in the management of psychiatric symptoms, since these vary significantly among the patients according to their phenotype. For example, one can design longitudinal studies using biological samples collected from living patients prior to and after a certain period of medication. Results may provide the possibility of discovering a set of biomarker candidates to be evaluated prior to the initiation of the treatment that could indicate whether a given treatment is likely to be successful or not for each particular patient. Obviously, such panel of biomarkers must be validated in large cohorts, established from samples collected in a noninvasive manner and employing reliable analytical platforms.

Biomarkers to the stratification of patients are also needed [4]. Schizophrenia, for instance, encompasses an umbrella of disorders which may be the result of the dysfunction of distinct molecular mechanisms triggered by environmental factors that will result, at the end of the day, in the same condition. For example, some patients might develop schizophrenia due to metabolic disturbances, while others 
might develop it due to inflammatory dysfunctions. In these cases, a genetic predisposition seems to play a pivotal role, considering the estimated heritability of schizophrenia in 80 to $85 \%$ [5], but acting through different molecular pathways. There are still those patients which suffered a very heavy pressure of the environment, which may have a more definitive part in the establishment of the disease than genetics [6]. In any case, each patient will have his/her particular molecular type of schizophrenia due to their distinct phenotypes. Thus patients must be categorized-or stratified-in a way that distinct treatments are administered in a tailor-made fashion. Such characterization could be done by determining molecular biomarkers to each type of patient. This solution would enhance the treatment, since each patient would be treated according to the type of molecular dysfunction he/she has or according to their phenotype. It has been observed a long time ago that the strategy of treating all patients with one kind of treatment does not seem to be the way to recover patients and place them back to society. Although schizophrenia was used as an example here, this strategy would be applicable for other psychotic and affective disorders.

Last but not least, it is important to be aware of how the concept of biomarker has been used. A biomarker is a measurable characteristic (i.e., molecule, physical structure or observation) assessed by a validated analytical platform which can indicate unequivocally a particular disease or physiological state of an organism or even the positive/negative response of such organism to a given treatment. The great majority of the data which has been published in the field of biomarkers do not fulfill this definition. Therefore, most of what we know so far are biomarker candidates or potential biomarkers only [7]. It is necessary to consider the different types of biomarkers proposed by the USA Food and Drug Administration (FDA) such as exploratory biomarkers, probably valid biomarkers, and known valid biomarkers when performing biomarker studies [8].

This special issue of Disease Markers approaches which is the current situation regarding the discovery of biomarkers to psychiatric disorders such as schizophrenia (Weickert et al. 2013), depression (Labermaier et al. 2013, Carboni 2013), posttraumatic stress disorder (Schmidt et al. 2013), and autism spectrum disorders (Voineagu and Yoo, 2013). I hope that the information presented in this edition may be useful to generate validation studies, develop trustworthy analytical platforms, launch new lines of research towards personalized medicine studies in order to approximate scientific studies to solutions clinically applicable, or yet to approximate the bench to the bedside.

Daniel Martins-de-Souza disorder in a changing professional environment," Journal of Mental Health, vol. 19, no. 4, pp. 337-351, 2010.

[3] E. Schwarz, R. Izmailov, M. Spain et al., "Validation of a bloodbased laboratory test to aid in the confirmation of a diagnosis of schizophrenia," Biomarker Insights, vol. 2010, no. 5, pp. 39-47, 2010.

[4] D. Martins-de-Souza, “Translational strategies to schizophrenia from a proteomic perspective," Translational Neuroscience, vol. 3, no. 3, pp. 300-302, 2012.

[5] A. G. Cardno and I. I. Gottesman, "Twin studies of schizophrenia: from bow-and-arrow concordances to star wars Mx and functional genomics," American Journal of Medical Genetics, vol. 97, no. 1, pp. 12-17, 2000.

[6] O. D. Howes, C. McDonald, M. Cannon, L. Arseneault, J. Boydell, and R. M. Murray, "Pathways to schizophrenia: the impact of environmental factors," International Journal of Neuropsychopharmacology, vol. 7, supplement 1, pp. S7-S13, 2004.

[7] D. Martins-de-Souza, "Is the word 'biomarker' being properly used by proteomics research in neuroscience?" The European Archives of Psychiatry and Clinical Neuroscience, vol. 260, no. 7, pp. 561-562, 2010.

[8] S. Bahn, E. Schwarz, L. W. Harris, D. Martins-de-Souza et al., "Biomarker blood tests for diagnosis and management of mental disorders: focus on schizophrenia," Revista de Psiquiatria Clínica, vol. 40, no. 1, article 7, 2013.

\section{References}

[1] A. Gonzalez-Pinto, M. Gutierrez, F. Mosquera et al., "First episode in bipolar disorder: misdiagnosis and psychotic symptoms," Journal of Affective Disorders, vol. 50, no. 1, pp. 41-44, 1998.

[2] J. C. Wakefield, "Misdiagnosing normality: psychiatry's failure to address the problem of false positive diagnoses of mental 


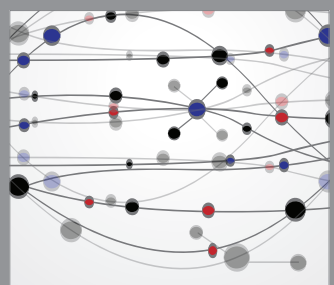

The Scientific World Journal
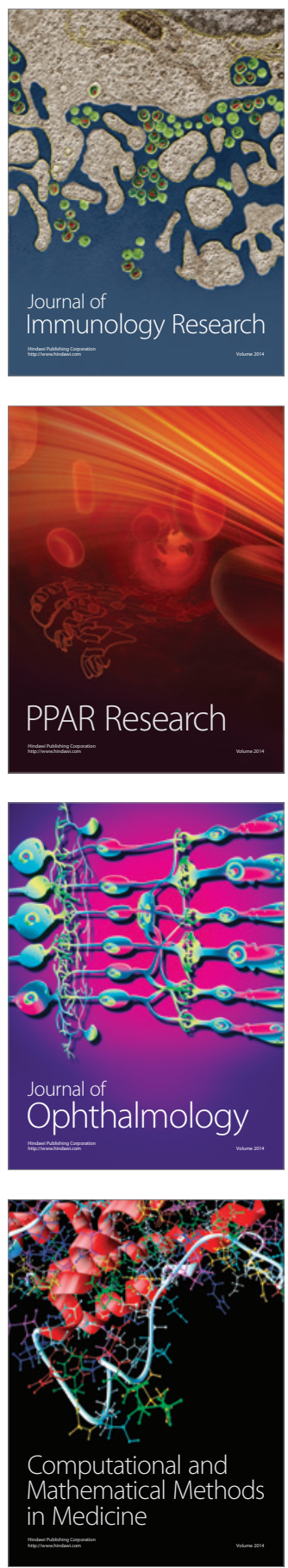

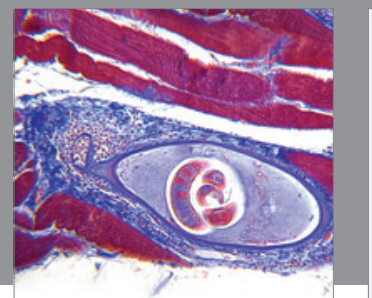

Gastroenterology

Research and Practice
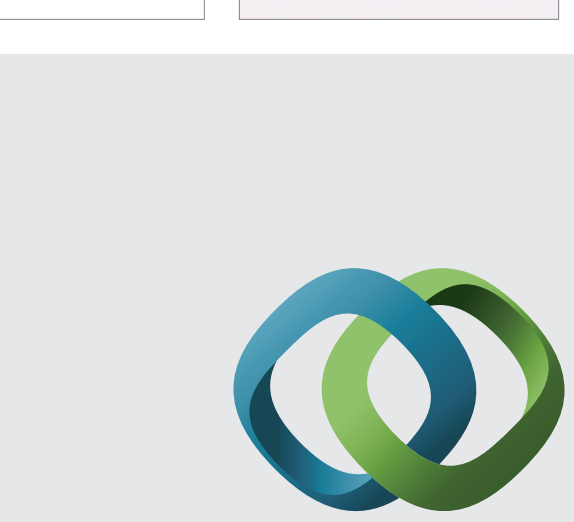

\section{Hindawi}

Submit your manuscripts at

http://www.hindawi.com
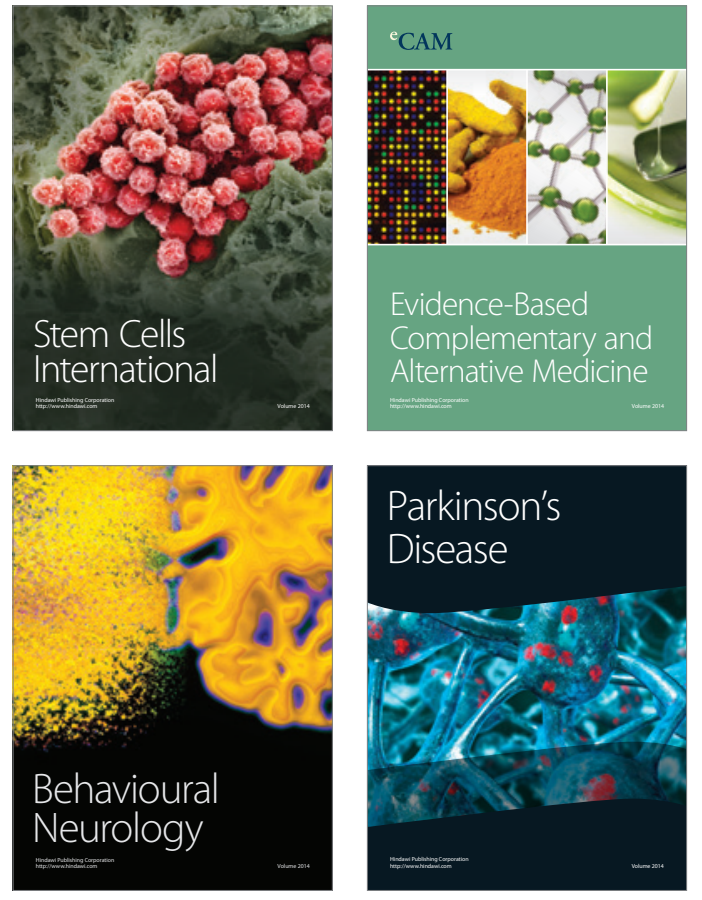
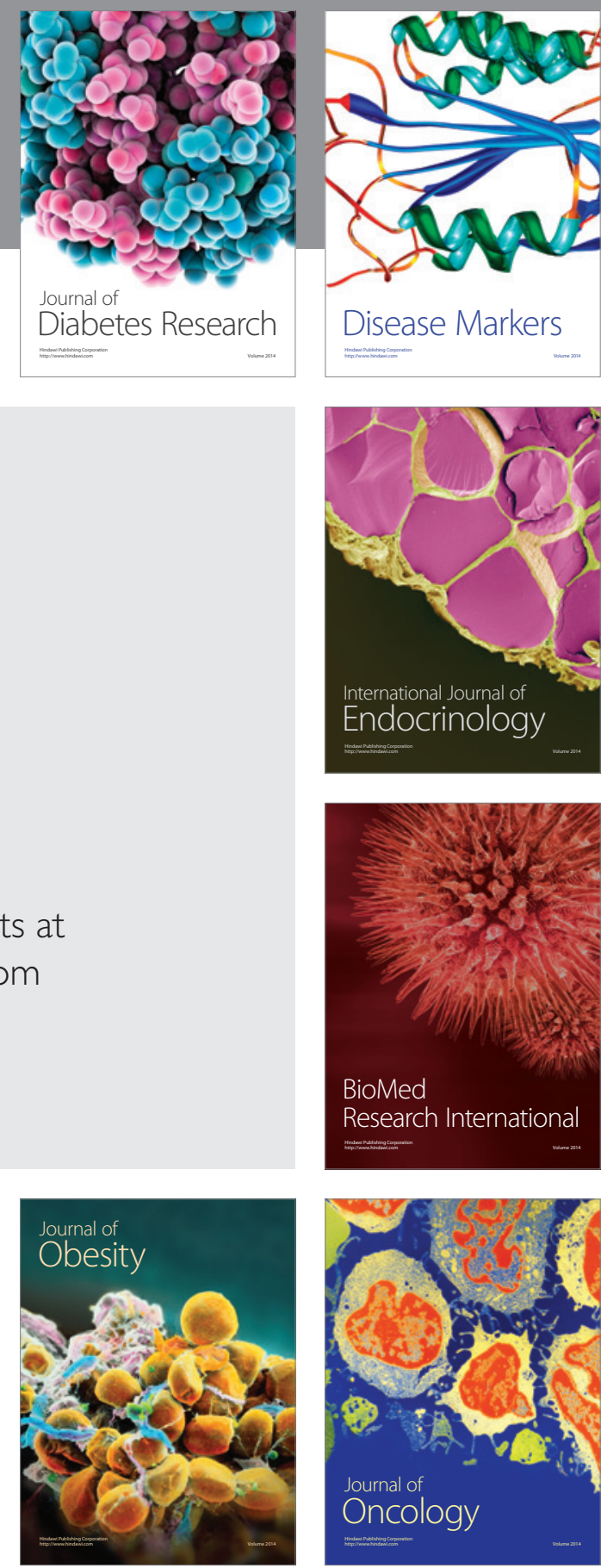

Disease Markers
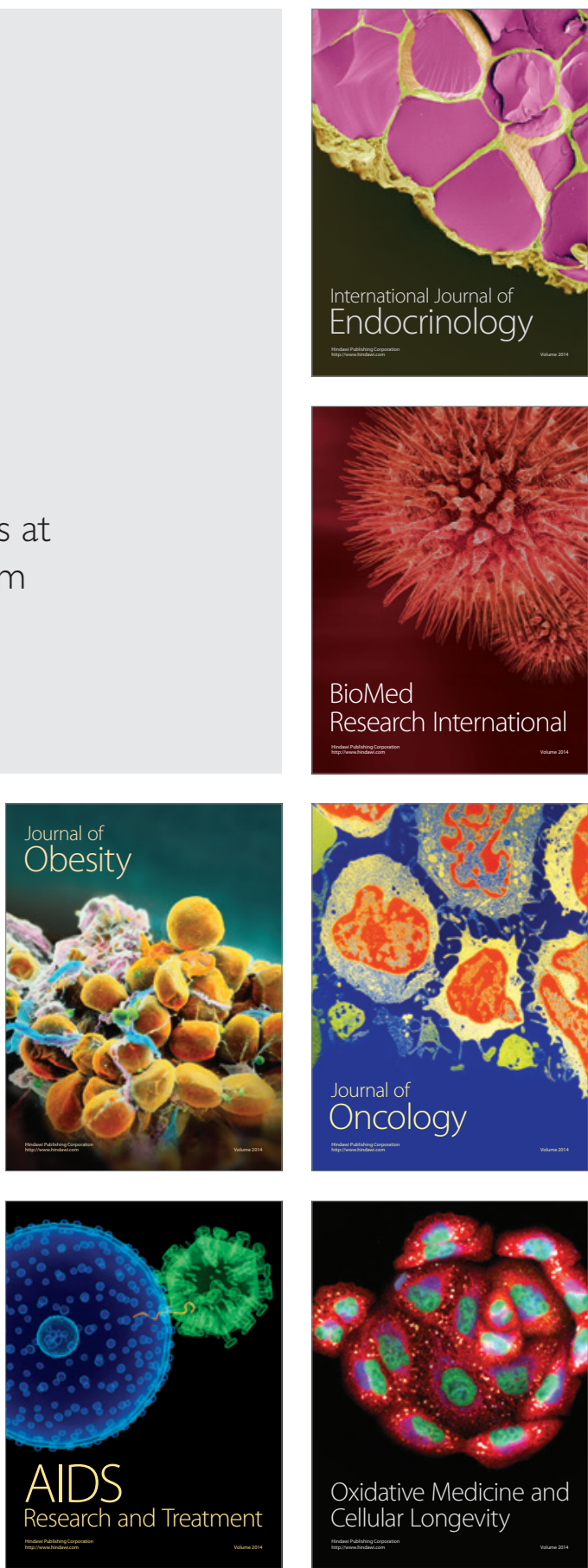